\title{
The spatiotemporal pattern of auditory cortical responses during verbal hallucinations
}

Citation for published version (APA):

van de Ven, V. G., Formisano, E., Roder, C. H., Prvulovic, D., Bittner, R. A., Dietz, M. G., Hubl, D., Dierks, T., Federspiel, A., Esposito, F., Di Salle, F., Jansma-Schmitt, B. M., Goebel, R., \& Linden, D. (2005). The spatiotemporal pattern of auditory cortical responses during verbal hallucinations. Neuroimage, 27(3), 644-655. https://doi.org/10.1016/j.neuroimage.2005.04.041

Document status and date:

Published: 01/01/2005

DOI:

10.1016/j.neuroimage.2005.04.041

Document Version:

Publisher's PDF, also known as Version of record

Document license:

Taverne

Please check the document version of this publication:

- A submitted manuscript is the version of the article upon submission and before peer-review. There can be important differences between the submitted version and the official published version of record.

People interested in the research are advised to contact the author for the final version of the publication, or visit the DOI to the publisher's website.

- The final author version and the galley proof are versions of the publication after peer review.

- The final published version features the final layout of the paper including the volume, issue and page numbers.

Link to publication

\footnotetext{
General rights rights.

- You may freely distribute the URL identifying the publication in the public portal. please follow below link for the End User Agreement:

www.umlib.nl/taverne-license

Take down policy

If you believe that this document breaches copyright please contact us at:

repository@maastrichtuniversity.nl

providing details and we will investigate your claim.
}

Copyright and moral rights for the publications made accessible in the public portal are retained by the authors and/or other copyright owners and it is a condition of accessing publications that users recognise and abide by the legal requirements associated with these

- Users may download and print one copy of any publication from the public portal for the purpose of private study or research.

- You may not further distribute the material or use it for any profit-making activity or commercial gain

If the publication is distributed under the terms of Article $25 \mathrm{fa}$ of the Dutch Copyright Act, indicated by the "Taverne" license above, 


\title{
NeuroImage
}

ELSEVIER

www.elsevier.com/locate/ynimg

NeuroImage 27 (2005) $644-655$

\section{The spatiotemporal pattern of auditory cortical responses during verbal hallucinations}

\author{
Vincent G. van de Ven, ${ }^{\mathrm{a}, \mathrm{b}, *}$ Elia Formisano, ${ }^{\mathrm{a}}$ Christian H. Röder, ${ }^{\mathrm{c}}$ David Prvulovic, ${ }^{\mathrm{b}}$ \\ Robert A. Bittner, ${ }^{\mathrm{b}}$ Matthias G. Dietz, ${ }^{\mathrm{d}}$ Daniela Hubl, ${ }^{\mathrm{e}}$ Thomas Dierks, ${ }^{\mathrm{e}}$ \\ Andrea Federspiel, ${ }^{\mathrm{e}}$ Fabrizio Esposito, ${ }^{\mathrm{f}}$ Francesco Di Salle, ${ }^{\mathrm{f}, \mathrm{g}}$ Bernadette Jansma, ${ }^{\mathrm{a}}$ \\ Rainer Goebel, ${ }^{\mathrm{a}}$ and David E.J. Linden ${ }^{\mathrm{b}, \mathrm{h}, \mathrm{i}}$ \\ a Department of Cognitive Neuroscience, Faculty of Psychology, University Maastricht, The Netherlands \\ ${ }^{\mathrm{b}}$ Laboratory for Neurophysiology and Neuroimaging, Department of Psychiatry, Johann Wolfgang Goethe-University, Germany \\ ${ }^{\mathrm{c}}$ Department of Psychiatry, Erasmus MC, Rotterdam, The Netherlands \\ ${ }^{\mathrm{d} D e p a r t m e n t}$ of Psychiatry, Markus Hospital, Frankfurt am Main, Germany \\ ${ }^{\mathrm{e}}$ Department of Psychiatric Neurophysiology, University Hospital of Clinical Psychiatry, University of Bern, Switzerland \\ ${ }^{\mathrm{f}}$ Department of Neuroradiology, Università Federico II, Naples, Italy \\ ${ }^{\mathrm{g}}$ Department of Neurosciences, University of Pisa, Italy \\ ${ }^{\mathrm{h}}$ Max Planck Institute for Brain Research, Department of Neurophysiology, Frankfurt am Main, Germany \\ ${ }^{\mathrm{i} S c h o o l}$ of Psychology, University of Wales, Bangor, UK
}

Received 8 December 2004; revised 11 March 2005; accepted 25 April 2005

Available online 22 June 2005

Functional magnetic resonance imaging (fMRI) studies can provide insight into the neural correlates of hallucinations. Commonly, such studies require self-reports about the timing of the hallucination events. While many studies have found activity in higher-order sensory cortical areas, only a few have demonstrated activity of the primary auditory cortex during auditory verbal hallucinations. In this case, using self-reports as a model of brain activity may not be sensitive enough to capture all neurophysiological signals related to hallucinations. We used spatial independent component analysis (sICA) to extract the activity patterns associated with auditory verbal hallucinations in six schizophrenia patients. SICA decomposes the functional data set into a set of spatial maps without the use of any input function. The resulting activity patterns from auditory and sensorimotor components were further analyzed in a single-subject fashion using a visualization tool that allows for easy inspection of the variability of regional brain responses. We found bilateral auditory cortex activity, including Heschl's gyrus, during hallucinations of one patient, and unilateral auditory cortex activity in two more patients. The associated time courses showed a large variability in the shape, amplitude, and time of onset relative to the self-reports. However, the average of the time courses during hallucinations showed a clear

* Corresponding author. Department of Cognitive Neuroscience, Faculty of Psychology, Universiteit Maastricht, PO Box 616, 6200 MD, Maastricht, The Netherlands.

E-mail address: v.vandeven@psychology.unimaas.nl

(V.G. van de Ven).

Available online on ScienceDirect (www.sciencedirect.com). association with this clinical phenomenon. We suggest that detection of this activity may be facilitated by examining hallucination epochs of sufficient length, in combination with a data-driven approach. (c) 2005 Elsevier Inc. All rights reserved.

Keywords: fMRI; Auditory hallucinations; Spatial ICA; Auditory cortex; Schizophrenia; BOLD

\section{Introduction}

Auditory verbal hallucinations (AVH), the perception of voices without the presence of an external correlate, represent one of the most baffling symptoms of psychiatric disorders and are a core symptom in the diagnosis of schizophrenia (American Psychiatric Association, 1994). Functional neuroimaging has made major contributions to the understanding of brain activity that underlies hallucinations. Modality- and content-specific brain activity has been observed in higher-order sensory areas during auditory (Silbersweig et al., 1995) as well as visual hallucinations (Ffytche et al., 1998), and early sensory areas have also been implicated (Woodruff et al., 1997). In particular, the primary auditory cortex (PAC) of the dominant hemisphere was found to be active during AVH (Dierks et al., 1999), and the lateralization of the activity suggests the involvement of language-related processes in AVH (Stephane et al., 2001), which is furthermore supported by alterations in white matter fiber tracts connecting language-related areas in hallucinating schizophrenia patients (Hubl et al., 2004). 
Activity in PAC is not found during the internal generation of an auditory image of spoken text (Shergill et al., 2001) or music (Halpern and Zatorre, 1999). Mental imagery is typically distinguished from the experience of hallucinations in terms of vividness and degree of control that one can exert upon the percept. The functional dissociation of activity patterns in the early auditory perception system between hallucinations and auditory mental imagery might thus be reflected in the increased vividness and lack of subjective control of AVH.

The finding of PAC activity during AVH, however, has remained controversial (Shergill et al., 2000), with only few replications (Bentaleb et al., 2002; Lennox et al., 2000). It has been argued that the measurement design used in these fMRI studies may have been a confounding factor that produced the PAC activity during hallucinations (Shergill et al., 2000). Most fMRI studies apply a functional measurement sequence that has short delays between consecutive whole-brain measurements. Thus, the broadband sound of the EPI sequence may function as an acoustic mask for the ongoing activity in the auditory cortex (Bandettini et al., 1998), which may influence the activity of PAC in a transient, unexpected manner. At the same time, the overt signaling of the timing of the hallucinations may confound the analysis. To counteract these methodological issues, Shergill et al. (2000) used a design where single-volume measurements were separated by time intervals ranging between 30 and $60 \mathrm{~s}$ (random sampling design), and where the patients reported on their hallucinations after each measurement. The authors did not find PAC activity associated with hallucinations and argued that the PAC activity found by others may have been modulated by scanner noise or hallucination-related self-reports. However, the potential benefit of the random sampling design is offset by a loss of temporal resolution and sensitivity.

Previous studies of hallucinations relied on correlation analysis (Bandettini et al., 1993) to identify signals of interest, which typically implies that a sequence of self-reports serves as a model for brain activity, which is applied to the data set in a voxel-pervoxel manner. This model, however, may not be ideal to trace brain activity related to hallucinations. For example, some cortical areas may show an onset of activity that precedes the button press by several seconds (Ffytche et al., 1998; Lennox et al., 1999). Moreover, the neural signatures of hallucinations may exhibit a much more complex variability than the responses to externally presented stimuli in experimental paradigms, for which a precise input function can be defined. At the same time, potential relations between the voxel time courses (e.g., functional connectivity elements in the data) are ignored.

In the current study we used spatial independent component analysis (sICA; McKeown et al., 1998a) to investigate the cortical activity patterns during AVH. SICA is a multivariate, data-driven method that does not rely on a predefined model of brain activity. Instead, sICA estimates a set of spatial source components from the observed data, which are characterized by maximum spatial independence between components (McKeown et al., 1998a). For neurophysiologically interesting signals, high voxel values tend to cluster in space (Formisano et al., 2002; Van de Ven et al., 2004). This approach has proven to be useful in separating spatial networks of activity that are related to neurophysiological events of interest from activity patterns that are related to other sources, such as head movements. SICA is able to separate networks of activity that are related to the random presentation of acoustic stimuli of varying duration (Gu et al., 2001), which makes it very suitable for the analysis of hallucinations. At the same time, sICA has been used to characterize deviating patterns of functional coupling in auditory cortex in schizophrenia (Calhoun et al., 2004). The temporal variability of the activity patterns of the obtained spatial maps can be further analyzed using the BOLD-image plot (Duann et al., 2002). This visualization tool allows for the investigation of single trial responses and aids the further interpretation of the extracted activity patterns.

We investigated activity in primary and secondary auditory cortex during AVH in schizophrenia using sICA and BOLD-image plots. Data of the same patients listening to acoustic stimuli were analyzed in the same way as the hallucination data. We demonstrate hallucination-related activity in auditory cortex, including Heschl's gyrus, in a number of patients. A large variability of the shape and timing of the cortical response during hallucinations compromises its detection by conventional correlation analysis, in which case sICA can complement the analysis.

\section{Methods}

Subjects

Six paranoid schizophrenia patients were recruited from the participating hospitals. Table 1 lists the age, sex, and medication of each patient at the time of measurement. Diagnosis was based on

Table 1

Demographic, medication, and measurement parameters of the hallucination $(\mathrm{H})$ and acoustic stimulation $(\mathrm{S})$ measurements of the six patients

\begin{tabular}{|c|c|c|c|c|c|c|c|c|c|}
\hline \multirow[t]{2}{*}{ Patient } & \multirow[t]{2}{*}{ Age (years) } & \multirow[t]{2}{*}{ Sex } & \multirow[t]{2}{*}{ Medication } & \multicolumn{6}{|c|}{ Measurement parameters } \\
\hline & & & & Session & $\mathrm{TR} / \mathrm{TE}(\mathrm{ms})$ & Nvols & Nslice & FOV $(\mathrm{mm})$ & Pixel size $(\mathrm{mm})$ \\
\hline \multirow[t]{2}{*}{ A } & 31 & $\mathrm{~m}$ & Bromperidol & $\mathrm{H}$ & $4000 / 60$ & 128 & 16 & $210 \times 210$ & $3.59 \times 3.59 \times 5$ \\
\hline & & & & S & $4000 / 60$ & 128 & 16 & $210 \times 210$ & $3.59 \times 3.59 \times 5$ \\
\hline \multirow[t]{2}{*}{ B } & 23 & $\mathrm{~m}$ & Clozapine/fluphenazine & $\mathrm{H}$ & $4000 / 60$ & 128 & 16 & $200 \times 200$ & $3.75 \times 3.75 \times 5$ \\
\hline & & & & $\mathrm{S}$ & $4000 / 60$ & 128 & 16 & $200 \times 200$ & $3.75 \times 3.75 \times 5$ \\
\hline \multirow[t]{2}{*}{$\mathrm{C}$} & 34 & $\mathrm{f}$ & Olanzapine & $\mathrm{H}$ & $4000 / 60$ & 128 & 24 & $200 \times 200$ & $3.13 \times 3.13 \times 5$ \\
\hline & & & & S & $4000 / 60$ & 128 & 24 & $200 \times 200$ & $3.13 \times 3.13 \times 5$ \\
\hline \multirow[t]{2}{*}{$\mathrm{D}$} & 46 & $\mathrm{~m}$ & Haloperidol/levomepromazine/lorazepam & $\mathrm{H}$ & $2000 / 60$ & 300 & 16 & $230 \times 230$ & $1.56 \times 1.56 \times 5$ \\
\hline & & & & S & $2000 / 60$ & 300 & 16 & $230 \times 230$ & $1.56 \times 1.56 \times 5$ \\
\hline \multirow[t]{2}{*}{ E } & 40 & $\mathrm{f}$ & Haloperidol/diazepam & $\mathrm{H}$ & $2000 / 60$ & 210 & 16 & $240 \times 240$ & $1.56 \times 1.56 \times 5$ \\
\hline & & & & S & $2000 / 60$ & 170 & 16 & $240 \times 240$ & $1.56 \times 1.56 \times 5$ \\
\hline \multirow[t]{2}{*}{$\mathrm{F}$} & 46 & $\mathrm{~m}$ & Amisulpride & $\mathrm{H}$ & $2000 / 60$ & 250 & 16 & $200 \times 200$ & $1.56 \times 1.56 \times 5$ \\
\hline & & & & S & $2000 / 60$ & 250 & 16 & $200 \times 200$ & $1.56 \times 1.56 \times 5$ \\
\hline
\end{tabular}

$\mathrm{TR} / \mathrm{TE}=$ time of repetition/echo time; Nvols = number of volumes; Nslice = number of slices; FOV = field of view; $\mathrm{m}(\mathrm{f})=\mathrm{male}(\mathrm{female})$. 
DSM-IV criteria (American Psychiatric Association, 1994) and was assessed by the treating psychiatrists. All patients were righthanded. The patients suffered predominantly from auditory verbal hallucinations that consisted of running commentary with derogatory content and that were resistant to antipsychotic medication. After the aim and instruction of the study had been explained to the patients, written informed consent was obtained. The study was approved by the local ethics committees.

\section{Design}

The data were acquired in the course of two separate studies on auditory hallucinations, one of which was published previously (Dierks et al., 1999). Patients A, B, and C participated in a followup of this study (study 1). Patients D, E, and F participated in a second study (study 2), which differed from study 1 by using only tones to localize PAC, and by acquiring the functional data with a higher temporal resolution.

For each patient the measurements comprised two functional (one for auditory hallucinations and one for acoustic stimulation) and one anatomical measurement. During the hallucination measurement the patient was required to press a button upon the onset of an auditory hallucination, hold it pressed during the hallucination, and release the button when the hallucination subsided. The sequences of button presses were converted into a reference time course for correlation analysis by assigning the value one to each volume in which the patient had pressed the button and zero to all other volumes. The reference time course was corrected for the delayed hemodynamic response (Boynton et al., 1996). Acoustic stimulation measurements were conducted in order to functionally localize the auditory cortex in each individual patient. During the acoustic stimulation measurement the patient was required to passively listen to the presented stimuli, using a custom made, scanner-compatible acoustic presentation system. For the patients of study 1 the acoustic stimuli consisted of blocks of speech, reversed speech and tones $(2 \mathrm{kHz})$, lasted for $24 \mathrm{~s}(8$ volumes) each, and with interspersed blocks of rest of equal length. For the patients of study 2 acoustic stimuli comprised blocks of tones of $0.5,1,2$, and $4 \mathrm{kHz}$. Patients $\mathrm{D}$ and $\mathrm{F}$ were presented with blocks of tones that lasted for $20 \mathrm{~s}$ each (10 volumes) and were interspersed with blocks of rest of equal length, during which no stimuli were presented. For patient $\mathrm{E}$, the blocks of tones and rest had variable lengths. Reference time courses for acoustic stimulation were obtained in the same way as for the hallucination epochs.

All functional and anatomical images were acquired using a 1.5 $T$ Magnetom Vision (Siemens, Erlangen, Germany) whole-body tomograph, using a gradient-echo EPI sequence for the measurement of the blood-oxygen-level-dependent (BOLD) signal. Table 1 lists the imaging parameters for the different sessions. For each patient a T1-weighted anatomical volume (MP-RAGE: magnetization-prepared rapid acquisition gradient echo) was acquired (voxel size $=1 \mathrm{~mm}^{3}$; matrix $=256 \times 256 \mathrm{~mm}$ ), which lasted approximately $8 \mathrm{~min}$.

Analysis

Preprocessing and independent component analysis

Preprocessing, spatial standardization, and sICA of the images were performed using the BrainVoyager 4.8 software (www. brainvoyager.com). Preprocessing of the functional images in- cluded slice time correction and three-dimensional spatial standardization into the Talairach and Tournoux (1988) coordinate space with a resampled functional voxel size of $3 \times 3 \times 3 \mathrm{~mm}$. The time courses were then temporally filtered by removing linear trends and by high-pass filtering of $1 / 125 \mathrm{~Hz}$ per functional run. The individual anatomical data sets were used to create a cortical mask that tags the corresponding functional voxels, which were then used for further analysis (Formisano et al., 2004).

The functional data sets were individually analyzed using cortex-based spatial independent component analysis (Formisano et al., 2002, 2004), which uses the FastICA algorithm (Hyvärinen, 1999). The application of cortex-based sICA to fMRI data is described in detail elsewhere (Formisano et al., 2004). Briefly, ICA in its general form models the observed data as a linear mixture of a set of statistically independent (latent) source variables, the number of which is equal or less than the number of observed variables. Formally, for fMRI data analysis the spatial ICA model is represented by

$\mathbf{X}=\mathbf{M S}$

where $\mathbf{X}$ is the $m \times n$ observed data matrix ( $m=$ number of acquired scans, $n=$ number of voxels), $\mathbf{S}$ is the $p \times n$ source components matrix with $p$ components, and $\mathbf{M}$ is the $m \times p$ matrix that contains the weights of the contribution of $\mathbf{S}$ to the data. The size of the decomposition can be set maximally to the number of observed variables (i.e., $p \leq m$ ), where the reduction is often achieved using principal component analysis (McKeown et al., 1998b). Both the mixing matrix and the source matrix are initially unknown, and component estimation is commonly achieved by maximizing the statistical independence between the latent variables, which can be done using different algorithms and estimates for statistical independence (Hyvärinen, 1999). The estimation of the independent component matrix $\mathbf{C}$ is then obtained by

$\mathbf{C}=\mathrm{WX}$

where $\mathrm{W}$ is the pseudo-inverse of M. FastICA maximizes statistical independence by maximizing negentropy, which is a measure of non-gaussianity of the probability distribution (Hyvärinen, 1999). The independent components are modeled as spatially independent and are therefore commonly referred to as component maps. The voxel values of the component maps (the rows of $\mathbf{S}$ and $\mathbf{C}$ ) are the component weights, and the voxel weight of one component conveys no information about the voxel values of any other component map, thereby maximizing spatial independence. The component maps are assumed to be time invariant, i.e., the layout of the component maps is assumed to be the same during the whole measurement. The contribution of an independent component to the data may be different for each observed variable, and plotting the contribution of a component map to the consecutive volumes constitutes the component time course, as contained within the columns of $\mathrm{W}$ in Eq. (2).

Selecting the number of components that must be estimated from the data is an important but complicated issue. Different heuristics for setting the number of components for the decomposition have been used (e.g., retaining $99-100 \%$ of the variance of the initial data; Gu et al., 2001; McKeown et al., 1998a). More formal methods for choosing the number of components have been suggested in the literature (Calhoun et al., 2001). In this study, we used a series of decompositions with different numbers of components in order to assess the reliability of components of interest. The initial decomposition was set at $1 / 4$ times the number 
of volumes of the functional data set for each individual measurement (this number was chosen arbitrarily while keeping the number of components relatively low and retaining more than $99 \%$ of the variance). Two additional decompositions were performed afterwards, one with $1 / 3$ of the number of volumes as number of components, and one with 100 components for each data set, regardless of the number of volumes. The reduction of the initial dimension of the data set to the size of the decomposition was achieved through principal component analysis.

For visualization the voxel values of each component map were $Z$-scored and thresholded at an absolute $Z$-value of 3 and a cluster size of 6 suprathreshold functional voxels. The maps were then superimposed on the anatomical image.

\section{Selecting components of interest and BOLD-image plots}

Selection and analysis of components of interest (COI) was performed using custom software programmed in Matlab 6 (MathWorks, Inc.). In this study we focussed only on the auditory cortex and sensorimotor cortex contralateral to the hand of button press during hallucinations, and auditory cortex during acoustic stimulation. Our primary aim was to identify components of auditory cortex activity during hallucinations and to compare these components to those of sensorimotor cortex activation during hallucinations and auditory cortex activation during acoustic stimulation. COIs were selected using standardized spatial templates based on a probabilistic map (Rademacher et al., 2001) and standardized coordinates (Talairach and Tournoux, 1988) of the bilateral auditory and sensorimotor cortex, respectively. A selected component was labeled as COI when (1) there were no obvious signs of artifactual contributions such as head movements or suprathreshold $Z$-values in areas known to be prone to large measurement artifacts (e.g., the sinuses), and (2) clusters of suprathreshold voxels within the template areas were of 6 contiguous functional voxels or larger.

The temporal pattern of the hallucination episodes of the COIs was analyzed using BOLD-image plots (Duann et al., 2002), a visualization technique that is derived from the study of single trial variability of event-related potentials (Jung et al., 2001). These plots depict the temporal variability across different trials or episodes. The plots were obtained in the following manner. The spatial COI maps were sign-corrected by multiplying the map values with the sign of the correlation coefficient between the COI time course and the reference time course. To prevent spurious corrections, the procedure was only executed for correlations higher than $|0.1|$. Then, for each COI the time courses of the suprathreshold voxels were extracted from the initial data set for further analysis. Note that voxel selection was not restricted to those voxels that fell within the boundaries of the spatial templates but comprised all suprathreshold voxels within the COI map. Independent component maps can be considered as maps of functional connectivity (Van de Ven et al., 2004), where the voxel values represent the amount of shared information (time-point by time-point) between the voxel time courses. Therefore, we chose to extract voxel time courses from the whole map since this would give a more accurate representation of the temporal information contained within the component. The extracted time courses were temporally smoothed using a Gaussian kernel of full width at half maximum $[\mathrm{FWHM}]=2$ time points and were averaged to obtain the mean COI activity time course. This time course was divided by the mean level of activity of the extracted time courses to obtain units of percent BOLD signal change.
We extracted the BOLD signal from the COI activity time courses for each hallucination or acoustic stimulation episode. The time window for an episode was set from one volume prior to the onset of the event up to $24 \mathrm{~s}$ from the onset. This procedure was the same for all measurements. An episode was discarded if (1) the distance over time between the onset of a hallucination and the end of the previous hallucination was less than $12 \mathrm{~s}$, or (2) the maximum percent BOLD signal change within the trial window exceeded $|6| \%$, in order to avoid the contribution of motion artefacts and large vessels. The trials that survived these criteria were interpolated to units of $1 \mathrm{~s}$ using spline interpolation, converted to horizontal color bars with color codes corresponding to the percent signal change values, and were stacked in the plots (Duann et al., 2002). The mean trial time course was appended to the plot.

\section{Results}

\section{Independent component analysis}

Table 2 presents the descriptive statistics of the hallucination episodes, as reported by the patients through button press. The following results pertain to the first decomposition (see Methods section). For patients A, D, and E only one COI that encompassed primary and secondary auditory cortex was found (see Figs. 1, 2, and 3). More specifically, for the decomposition of patient A the COI comprised clusters in left Heschl's gyrus, for patient D it comprised bilateral Heschl's gyrus, and for patient E right anterior and lateral Heschl's gyrus and posterior superior temporal gyrus. For patient F, several COIs that contained clusters of high absolute $Z$-values in auditory cortex were found, of which only one component correlated moderately with the button press response. For patients $\mathrm{B}$ and $\mathrm{C}$ no auditory cortex COI was detected. A COI for sensorimotor cortex contralateral to the hand of button press was found in five out of six patients, with the exception of patient C. For each of the acoustic stimulation measurements one COI comprising bilateral auditory cortex was detected (Fig. 4). Table 3 lists the spatial coordinates and cluster sizes of the suprathreshold voxels in the template areas for each COI.

We verified that the selected COIs correctly reflected the BOLD activity related to hallucinations and acoustic stimulation by correlating the COI activity time courses with the hallucination or acoustic stimulation reference models. Most correlations were significant $(P<0.05$; see Table 4$)$, suggesting that the COIs did indeed reflect the BOLD responses related to hallucinations or acoustic stimulation.

Table 2

Descriptive statistics of the hallucination self-reports of the patients

\begin{tabular}{|c|c|c|c|c|c|c|}
\hline \multirow[t]{2}{*}{ Patient } & \multirow{2}{*}{$\begin{array}{l}\text { Hand of } \\
\text { button } \\
\text { press }\end{array}$} & \multirow{2}{*}{$\begin{array}{l}\text { No. of } \\
\text { hallucination } \\
\text { epochs }\end{array}$} & \multirow[t]{2}{*}{$\mathrm{TR}$} & \multicolumn{3}{|c|}{ Duration of epochs (in volumes) } \\
\hline & & & & Range & Mean (SD) & $\mathrm{h} / \mathrm{nvols}$ \\
\hline A & Left & 10 & 4 & $2-10$ & $5.9(3.0)$ & 0.46 \\
\hline $\mathrm{B}$ & Left & 9 & 4 & $1-6$ & $3.2(1.6)$ & 0.23 \\
\hline $\mathrm{C}$ & Left & 23 & 4 & $1-5$ & $1.5(0.9)$ & 0.28 \\
\hline $\mathrm{D}$ & Left & 22 & 2 & $1-23$ & $6.4(5.0)$ & 0.48 \\
\hline $\mathrm{E}$ & Right & 9 & 2 & $4-14$ & $6.8(2.9)$ & 0.30 \\
\hline $\mathrm{F}$ & Right & 54 & 2 & $1-4$ & $1.9(0.6)$ & 0.42 \\
\hline
\end{tabular}

$\mathrm{TR}=$ time of repetition (in seconds); $\mathrm{h} / \mathrm{nvols}=$ proportion of measured volumes associated with hallucination self-report. 


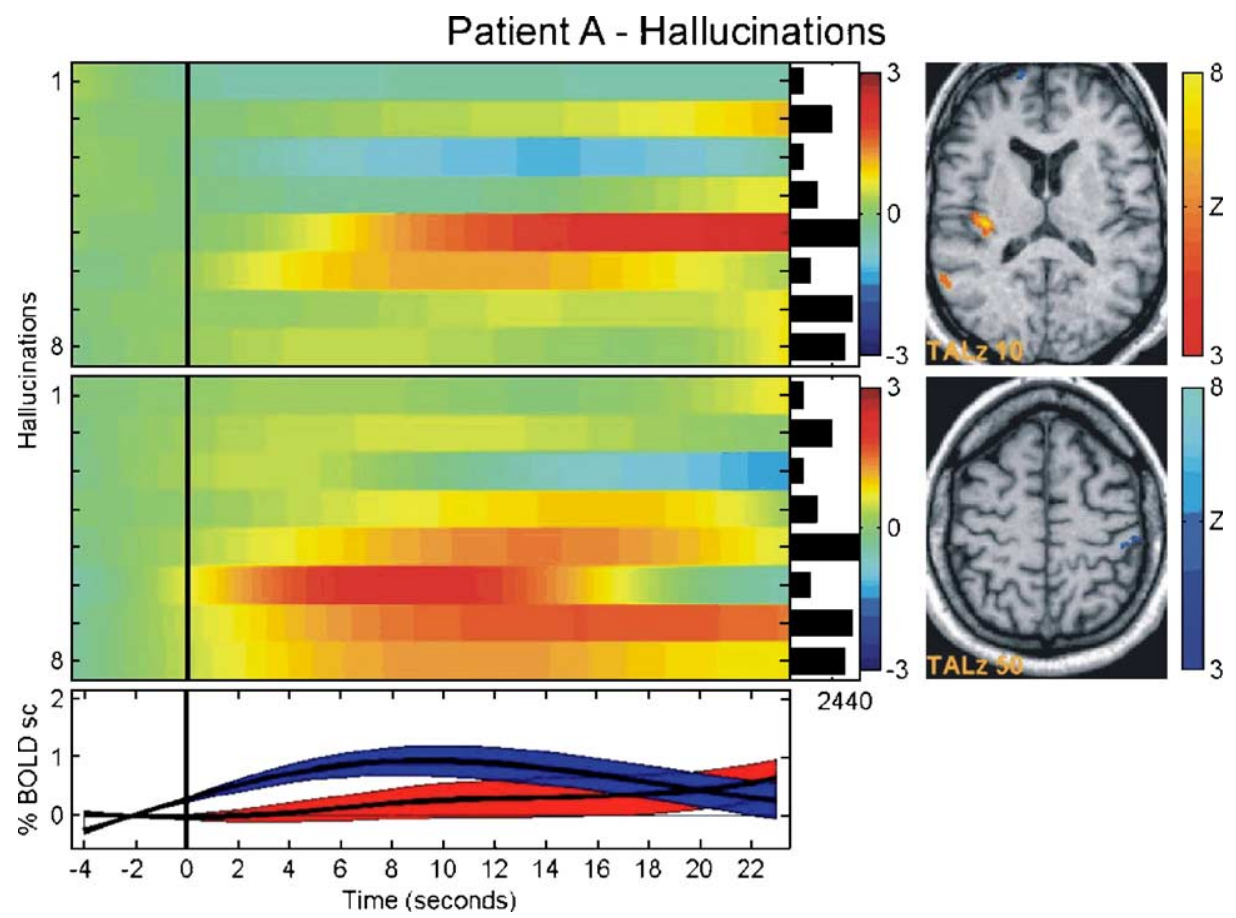

Fig. 1. BOLD-image plot of the auditory and sensorimotor components of interest during hallucinations of patient A. From left to right for each row: (1) the plots with horizontal color bars depict the percent BOLD signal change (\% BOLD sc) for eight hallucination episodes (BOLD-image plot [upper row: auditory component; lower row: sensorimotor component]); (2) attached to the right of each BOLD-image plot is the bar plot for the lengths of each hallucination episode (time in seconds); (3) attached to the right of the bar plot is the color legend for the BOLD-image plot; (4) on the utmost right the component map is shown superimposed on the anatomical image (left is left; the transverse coordinate in standardized space [TALz] in orange). The $Z$-values of the component map for auditory cortex are shown in red-yellow color scale and the map values for sensorimotor cortex in dark blue-light blue, each thresholded at $Z=3$. Below the BOLD-image plots the averaged episode time courses, with 1 standard error of the mean, are shown for the auditory (red) and sensorimotor (blue) components. The plots show that, despite a wide variation in onset, peak and overall shape of the BOLD signals over episodes, on average there is an increase in left auditory cortex.

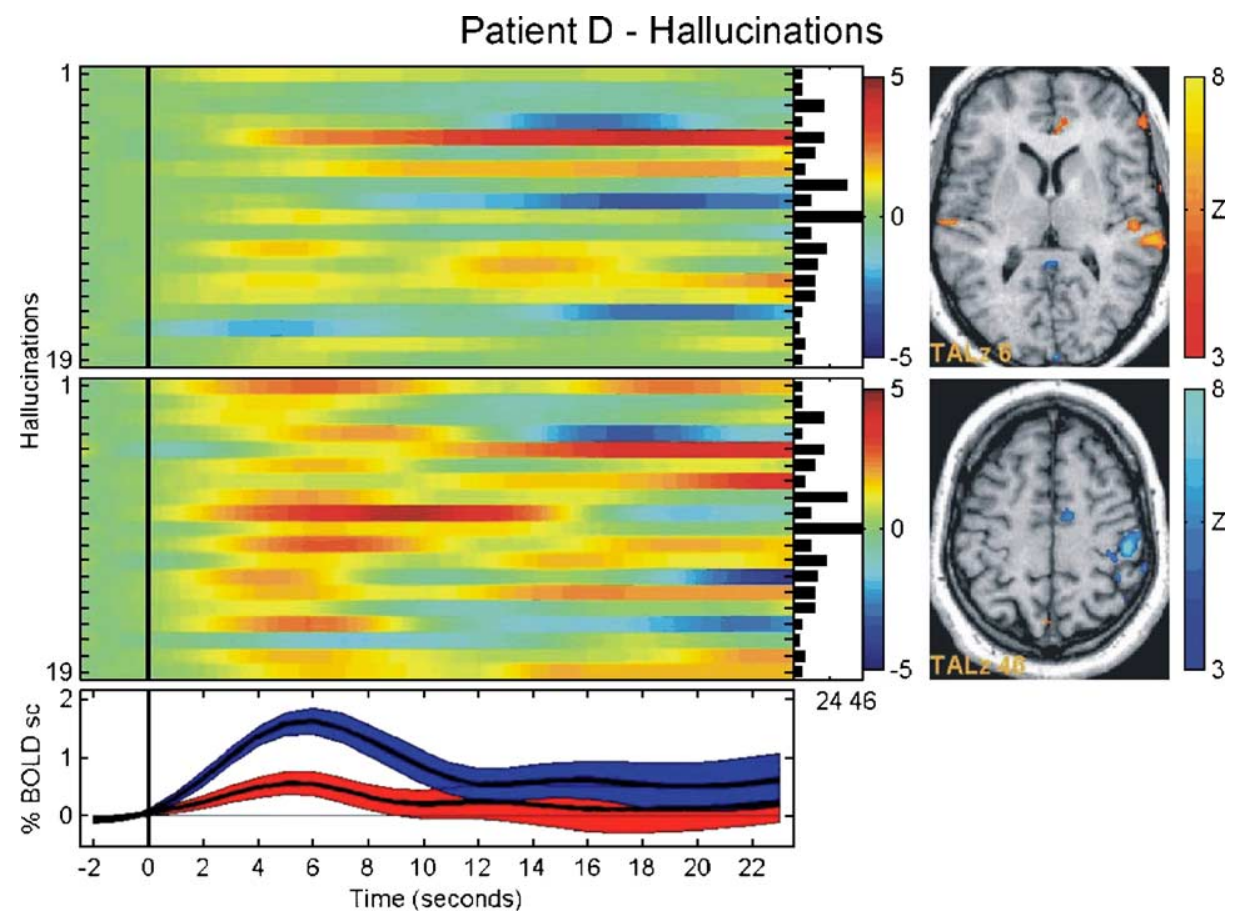

Fig. 2. BOLD-image plot of the auditory and sensorimotor components of interest during hallucinations of patient D. The plots show that for bilateral primary auditory cortex the average BOLD episode response increases in the early phase of the hallucinations. For further detail, see Fig. 1. 


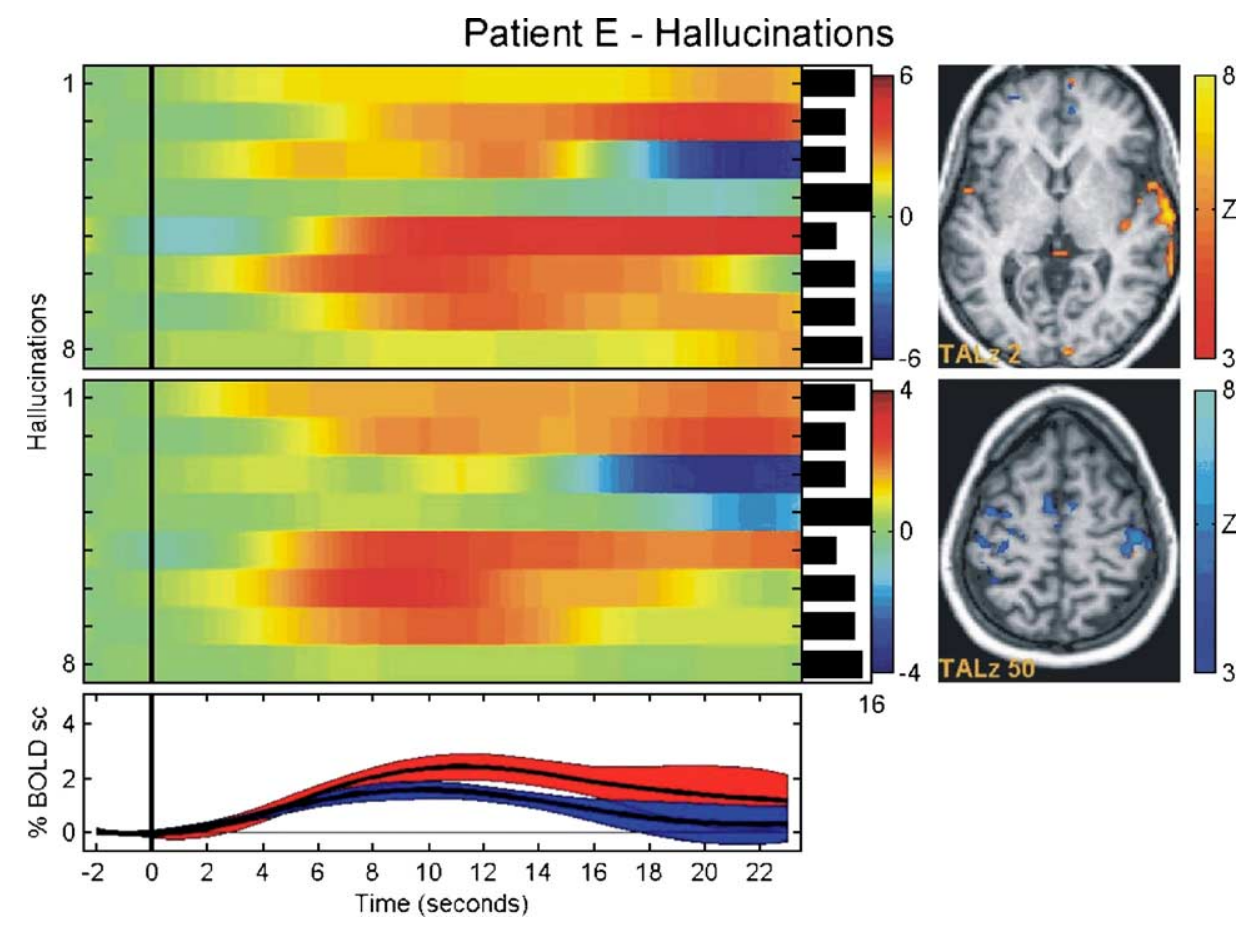

Fig. 3. BOLD-image plot of the auditory and sensorimotor components of interest during hallucinations of patient E. The plots show that for right primary auditory cortex the average BOLD episode response increases in the early phase of the hallucinations. For further detail, see Fig. 1.

\section{BOLD-image plots}

The BOLD-image plots of the hallucination measurements for patients A, D, and E are depicted in Figs. 1, 2, and 3. In each figure the bar plots to the right side of the BOLD-image plots show the duration of each button press response. The panel below the BOLD-image plots shows the averages of the episode-related BOLD signals, with the area of one standard error of the mean around the average time courses (red for auditory; blue for sensorimotor). A transverse image of the component map superimposed on the anatomical image is depicted on the right of each BOLD-image plot (red-orange for auditory; blue-green for sensorimotor). The BOLD-image plots of the hallucinations reveal a large variability in the time of onset, peak, and shape of the single episode BOLD responses within the auditory COIs. However, the averages of the single episode responses indicate that BOLD activity increased during episodes of hallucinations. Fig. 4 shows the BOLD-image plots for the acoustic stimulation sessions of the six patients.

In a next step, we descriptively examined whether hallucination parameters were related to the detection of an auditory COI during hallucinations. To this end, we plotted the mean duration of hallucinations, the percentage of self-reports during the measurement, and the number of reported hallucinations for the six patients (Fig. 5). The average duration of hallucinations seemed to be most strongly related to the detection of an auditory COI. The scores on this parameter were the lowest for patient $\mathrm{C}$, for whom no auditory or sensorimotor COI was detected.

\section{Post hoc comparisons}

When the three decompositions (number of components: a quarter and a third of number of volumes, and 100 regardless of number of volumes) were compared, a few differences emerged. For the additional decompositions the auditory COI during hallucinations of patient A comprised bilateral Heschl's gyrus. Clusters in bilateral Heschl's gyrus were found for the initial decomposition when the $Z$-threshold for visualization was lowered. The additional decompositions of the hallucination measurement of patient $\mathrm{F}$ revealed components with suprathreshold clusters in left Heschl's gyrus but these were not significantly correlated with the reference time course. Because of these marked differences between the decompositions it was concluded that the hallucination measurement of patient $\mathrm{F}$ does not comprise specific auditory cortex activity during hallucinations. All other results of the initial decompositions were replicated in the additional decompositions. Fig. 6A shows the amount of overlap of the auditory COIs of hallucinations between the different decompositions of patients $\mathrm{A}$, $\mathrm{D}$, and $\mathrm{E}$ (see Table 5 for spatial correlations between COIs of the different decompositions).

Cortex-based correlation analysis of the hallucination data sets using delayed and convolved (Boynton et al., 1996) reference time courses of the button presses showed that activity increased in primary and secondary auditory cortex during hallucinations for patients $\mathrm{A}$ and $\mathrm{E}$ (Fig. 6B), but not for patient D. Correlation maps were converted to a $Z$-distribution, and the correlation threshold was set in such a way that the number of significantly active voxels equaled the total number of suprathreshold voxels within the auditory and sensorimotor COI. A region of interest analysis of left and right Heschl's gyrus of patient $\mathrm{D}$, based on anatomical landmarks, verified that bilateral putative PAC is active during $\mathrm{AVH}$ as well as acoustic stimulation (Fig. 7).

Finally, estimation of event-related changes in BOLD signal for the suprathreshold clusters in left and right Heschl's gyrus of each auditory COI during hallucinations showed that time courses from 

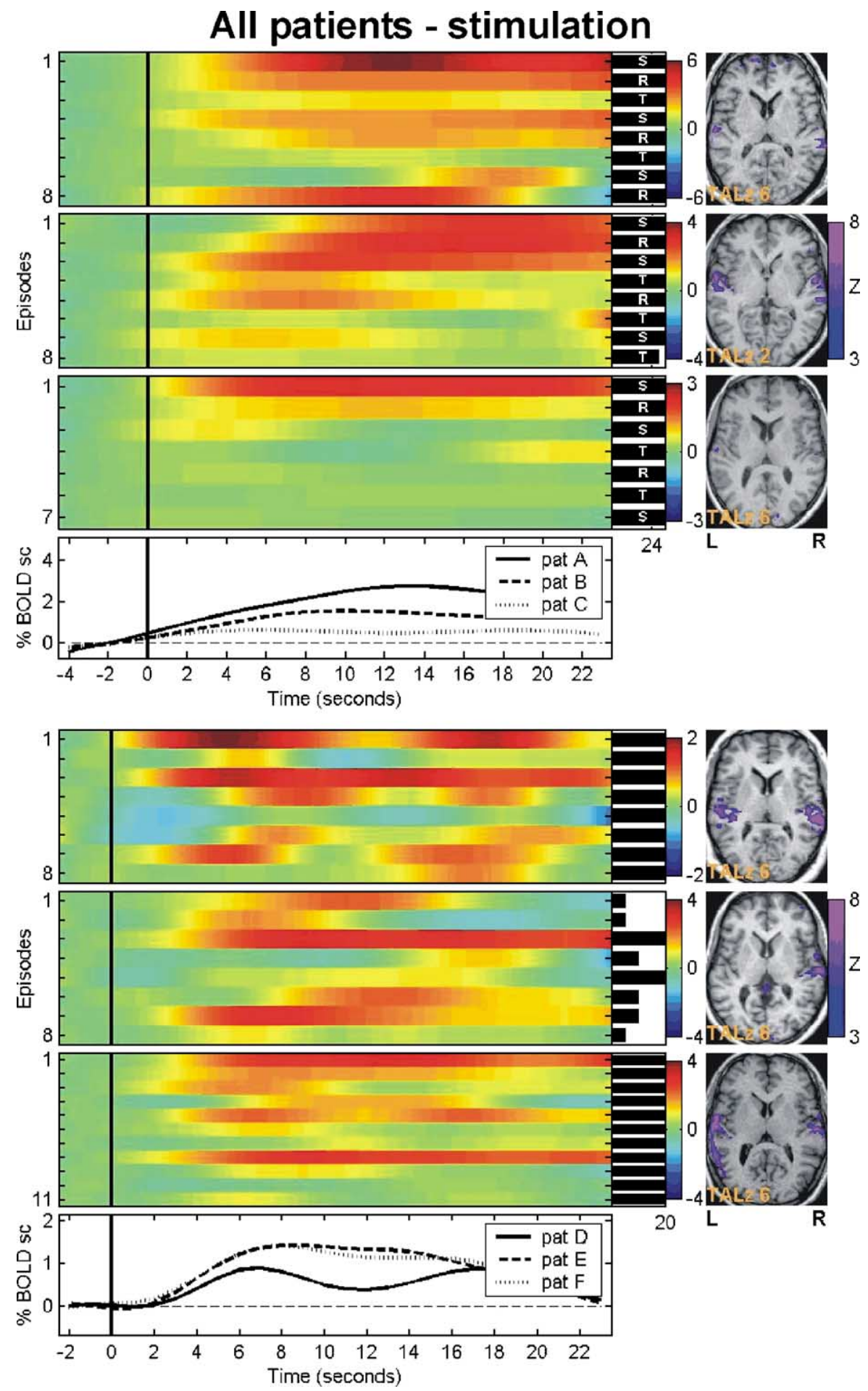

Fig. 4. BOLD-image plots of the auditory cortex during acoustic stimulation of the six patients. The plots are grouped for study 1 and study 2 (see text). For patients $\mathrm{A}, \mathrm{B}$, and $\mathrm{C}: \mathrm{S}=$ speech; $\mathrm{R}=$ reversed speech; $\mathrm{T}$ = tones. All acoustic stimuli for patients $\mathrm{D}, \mathrm{E}$, and $\mathrm{F}$ comprised tones. For further detail, see Fig. 1 .

these clusters reflected the average COI activity time course (Fig. 7).

\section{Discussion}

We analyzed data of six hallucinating schizophrenia patients using spatial independent component analysis (sICA) and charac- terized the activity in the left and right auditory cortex, including Heschl's gyrus, the site of primary auditory cortex (PAC) in humans (Formisano et al., 2003; Rademacher et al., 2001), during auditory verbal hallucinations (AVH) and acoustic stimulation. The hallucination measurements of three patients yielded components of interest (COIs) in both auditory cortex and sensorimotor cortex (related to the self-report by button press). Patient D showed hallucination-related activity in bilateral primary and secondary 
Table 3

Standardized spatial coordinates $[x, y, z]$ of the peak $Z$-value and cluster size $(\mathrm{mm})$ of the regions of interest in the obtained components of interest during hallucinations and acoustic stimulation

\begin{tabular}{|c|c|c|c|c|c|c|c|c|c|}
\hline \multirow[t]{2}{*}{ Patient } & \multirow[t]{2}{*}{$\mathrm{Sn}$} & \multirow[t]{2}{*}{$\mathrm{COI}$} & \multirow[t]{2}{*}{ Area+ } & \multicolumn{3}{|l|}{ Left } & \multicolumn{3}{|l|}{ Right } \\
\hline & & & & $x, y, z$ & $\max Z$ & size & $x, y, z$ & $\max Z$ & size \\
\hline \multirow[t]{6}{*}{ A } & $\mathrm{H}$ & $\mathrm{HG}$ & \multirow{4}{*}{ РTP } & $-37,-23,9$ & 7.3 & 621 & $56,-5,0$ & 4.9 & 378 \\
\hline & & & & $-52,-32,12$ & 5.1 & 459 & & & \\
\hline & & SMC & & & & & $44,-23,60$ & 5.7 & 459 \\
\hline & & & & & & & $44,-17,60$ & 7.7 & 270 \\
\hline & $\mathrm{S}$ & $\mathrm{HG}$ & \multirow{5}{*}{ STG } & $-61,-17,6$ & 11.9 & 540 & & & \\
\hline & & & & & & & $65,-35,9$ & 11.3 & 2430 \\
\hline \multirow[t]{3}{*}{ B } & $\mathrm{H}$ & $\mathrm{SMC}$ & & & & & $41,-17,42$ & 5.3 & 243 \\
\hline & $\mathrm{S}$ & $\mathrm{HG}$ & & $-64,-26,18$ & 20.8 & 2214 & $62,-11,9$ & 10.3 & 2889 \\
\hline & & & & $-61,-2,0$ & 8.9 & 3024 & & & \\
\hline $\mathrm{C}$ & $\mathrm{S}$ & $\mathrm{HG}$ & \multirow{5}{*}{ PTP } & $-64,-20,3$ & 4.2 & 405 & & & \\
\hline \multirow[t]{4}{*}{ D } & $\mathrm{H}$ & $\mathrm{HG}$ & & $-46,-23,24$ & 3.6 & 216 & $47,-20,6$ & 5.6 & 378 \\
\hline & & & & $-58,-23,24$ & 8.8 & 2862 & & & \\
\hline & & $\mathrm{SMC}$ & & & & & $38,-35,57$ & 14.6 & 5670 \\
\hline & $\mathrm{S}$ & $\mathrm{HG}$ & & $-52,-23,0$ & 9.5 & 5562 & $53,-32,6$ & 9.1 & 3834 \\
\hline \multirow[t]{5}{*}{$\mathrm{E}$} & $\mathrm{H}$ & $\mathrm{HG} / \mathrm{STG}$ & \multirow{9}{*}{ STG } & & & & $53,4,-6$ & 16.1 & 5076 \\
\hline & & SMC & & $-28,-20,63$ & 7.4 & 621 & & & \\
\hline & & & & $-46,-23,51$ & 4.2 & 189 & & & \\
\hline & $\mathrm{S}$ & $\mathrm{HG}$ & & $-46,-29,6$ & 3.4 & 216 & $41,-23,3$ & 4.5 & 513 \\
\hline & & & & & & & $53,4,-9$ & 16.4 & 3834 \\
\hline \multirow[t]{4}{*}{$\mathrm{F}$} & $\mathrm{H}$ & $\mathrm{SMC}$ & & $-31,-32,66$ & 4.9 & 297 & & & \\
\hline & & & & $-37,-32,42$ & 7.5 & 2511 & & & \\
\hline & $\mathrm{S}$ & HG/STG & & $-61,-8,9$ & 11.4 & 3996 & $59,-2,6$ & 4.9 & 243 \\
\hline & & & & & & & $44,-26,12$ & 7.9 & 1755 \\
\hline
\end{tabular}

Notes. $\mathrm{Sn}=$ session; $\operatorname{maxZ}=$ maximum $Z$-value; $\mathrm{SMC}=$ sensorimotor cortex; $\mathrm{HG}=$ Heschl's gyrus; $\mathrm{STG}=$ superior temporal gyrus; PTP = posterior temporal plane; $\mathrm{H}=$ hallucinations; $\mathrm{S}=$ acoustic stimulation; Area $+=$ additional area of suprathreshold $Z$-values that overlaps with the region of interest.

auditory cortex and two other patients only unilaterally (in the left hemisphere for patient $\mathrm{A}$, in the right for patient $\mathrm{E}$ ). These findings proved to hold with different number of components estimated, with the exception that for patient $\mathrm{A}$ the additional decompositions revealed clusters in bilateral PAC. COI activity time courses were extracted from the spatial maps, and the temporal variability of the activity time course was investigated using BOLD-image plots. The COI activity time courses showed a large variability in time of onset and amplitude across the various epochs. Nevertheless, all COI activity time courses of the auditory components showed an increase in activity during the hallucination episodes.

Table 4

Correlations between average BOLD time course obtained from the positive voxels of the component of interest and the button press/acoustic stimulation reference model

\begin{tabular}{llll}
\hline Patient & AVH & AS \\
\cline { 2 - 3 } & AC & SMC & AC \\
\hline A & $0.12^{\mathrm{a}}$ & 0.41 & 0.65 \\
B & - & $0.15^{\mathrm{a}}$ & 0.74 \\
C & - & - & 0.28 \\
D & 0.20 & 0.31 & 0.46 \\
E & 0.39 & 0.53 & 0.33 \\
F & - & $0.11^{\mathrm{a}}$ & 0.55 \\
\hline
\end{tabular}

Components of interest are auditory (AC) and sensorimotor (SMC) components obtained from the hallucination $(\mathrm{AVH})$ and acoustic stimulation (AS) sessions.

Notes. All $P$ 's $<0.01$, except.

${ }^{\text {a }} P<0.05$.

${ }^{\mathrm{b}}$ Not significant.
Activity in bilateral auditory cortex was found for all acoustic stimulation measurements. Although the auditory COIs of both the hallucination and acoustic stimulation included Heschl's gyrus, the COIs differed between the sessions in the size of the suprathreshold clusters and the presence of clusters outside of the auditory cortex. Taken together, these results indicate PAC activity during AVH (Bentaleb et al., 2002; Dierks et al., 1999; Lennox et al., 2000) in a number of patients but do not indicate a specific involvement of the dominant hemisphere (Dierks et al., 1999). Furthermore, the results show that data-driven analysis may complement hypothesis-driven analyses for complex and unpredictable phenomena such as hallucinations.

The hemispheric side of sensorimotor cortex activity due to the button presses did not seem to be related to the hemispheric side of the auditory cortex activity. This suggests that the auditory signal is not in some way influenced by sensorimotor activity, which is supported by the finding that the auditory and sensorimotor cortex activities were contained in two separate components. For patient $\mathrm{C}$ we failed to detect a sensorimotor COI related to the button presses. Post hoc correlation analysis revealed small clusters of activity in sensorimotor cortex only at a low threshold $(P<$ 0.0017, uncorrected), which may indicate that the overall functional contrast-to-noise in this subject was too low for activity associated with hallucinations to be detected.

Dierks et al. (1999) suggested that the activity in PAC represents the high degree of vividness of the hallucination that is commonly reported by the patients. This interpretation would be supported by a lack of activity in PAC during the generation and manipulation of an auditory verbal (Shergill et al., 2001) or musical (Halpern and Zatorre, 1999) mental image in healthy individuals 

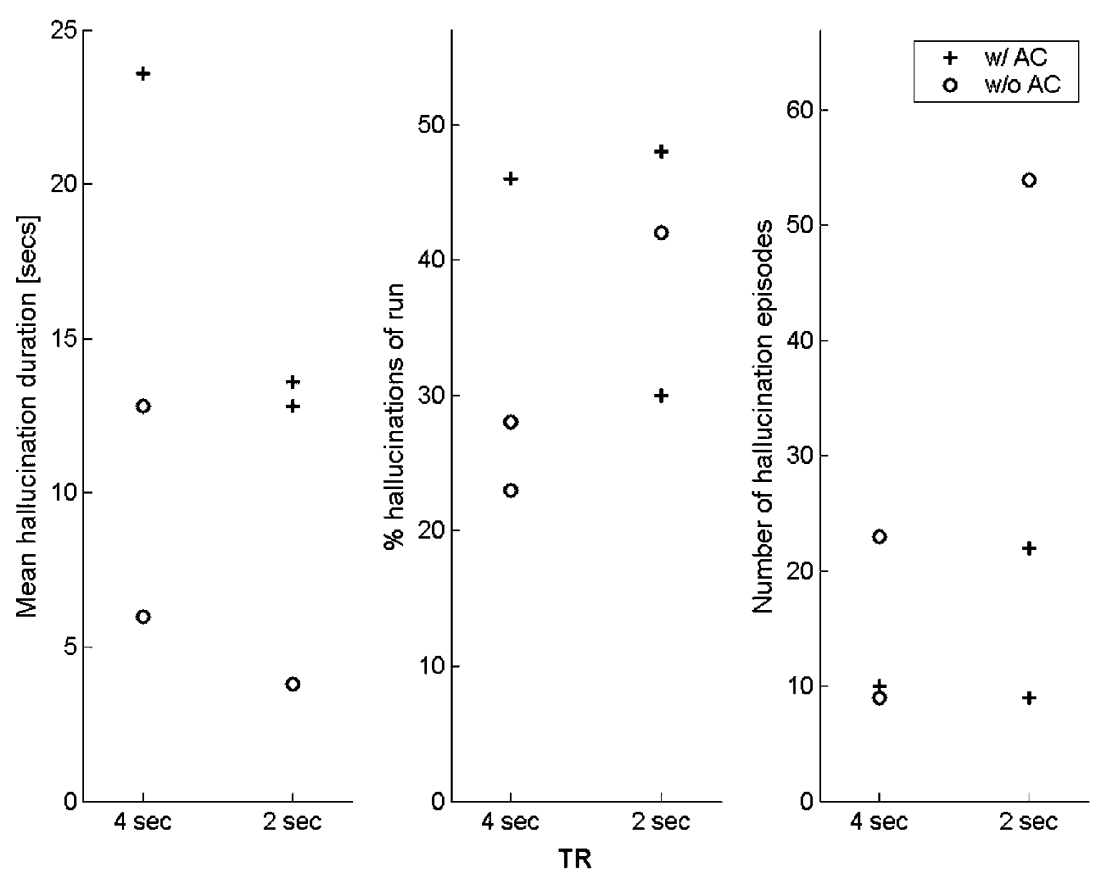

Fig. 5. From left to right: scatterplots for mean hallucination duration (in seconds), percentage of scanning time during which hallucinations were reported, and total number of hallucination episodes for the six patients. $\mathrm{TR}=$ repetition time; w/o AC = without detection of an auditory component.

because hallucinations are typically experienced as more vivid and real than mental images. The results of Engelien et al. (2000), who showed that a neurologic patient with lesions that comprised bilateral Heschl's gyrus could report the coarse characteristics of acoustic stimuli (e.g., on- and offset of a stimulus), but not their detailed features, conform to this view. Vivid recall of memorized sounds activated secondary, but not primary auditory cortex in healthy subjects (Wheeler et al., 2000), which may suggest that PAC activity during hallucinations is of a pathological nature. It is currently not known, however, whether similar increased activity in primary sensory cortex during imagery or memory recall in healthy subjects would also induce a misidentification of the source (internal vs. external) of the imagined or recalled item. However, the activity in auditory cortex during hallucinations may also reflect attentional responses to the presence of a hallucination because activity in PAC can be enhanced through attentional modulation (Jäncke et al., 1999). The saliency of an AVH, as well as the task instruction of monitoring the hallucination events, may thus have resulted in the temporary enhancement of cortical activity.

Our study shows that the on- and offset of auditory cortex activity might deviate considerably from the button press report. Sources of this variability may be found in patient compliance, task instruction, and complexity of auditory cortex activity. Firstly, the patients' self-reports themselves may have been inaccurate. The inherent difficulty to distinguish a hallucination from a real experience is a prominent characteristic of hallucinations as a clinical phenomenon (American Psychiatric Association, 1994), and hallucinations have been associated with a failure to monitor and identify the source of internally generated images (Blakemore et al., 2000). Although the patients reported that they could reliably detect their hallucinations, we could not verify their reported accuracy, or whether there was a bias towards reporting a hallucination in case of doubt. In addition, the activity of PAC may not be a universal characteristic of hallucination-related brain activity, reflecting the phenomenological variability of AVHs (Stephane et al., 2003). For example, highly detailed and vivid verbal hallucinations may recruit processing capacity of PAC to a larger extent than hallucinations where the voices are distant and the linguistic content is vague.

Secondly, the variability of the neural signal may be related to the explicit instruction to monitor and respond to the hallucination events. Attentional effects within auditory cortex (Jäncke et al., 1999) that are secondary to the hallucination may have altered the BOLD response with respect to the button presses.

Thirdly, the auditory cortical response to stimuli of varying duration has proven to be complex in healthy subjects (Harms and Melcher, 2003; Seifritz et al., 2002) as well as in the patients in this study. This complexity may increase further in the case of auditory percepts of a pathophysiological nature. The primary and secondary auditory cortex show anatomical (Hirayasu et al., 2002) and functional (Calhoun et al., 2004) abnormalities in schizophrenia patients, in comparison to healthy subjects, which may add to the complexity of the neural response of AVH.

Many of these difficulties are currently inherent to the study of hallucinations in general. In all of these cases a sequence of selfreports may prove to be an inaccurate model for hallucinationrelated brain activity. Because sICA does not depend on any predefined model of brain activity, it can complement conventional (hypothesis-driven) approaches in the attempt to identify the neural signal that is associated with AVH.

The detection of an auditory cortex COI during hallucinations seemed related to the average length of episodes of hallucinations. This finding would suggest that auditory cortex becomes active, or rather that its activity only becomes detectable with standard fMRI, if episodes of hallucinations last long enough. In line with previous suggestions (Dierks et al., 1999), the average length of hallucination episodes should be at least $10 \mathrm{~s}$ in order to be reliably detected. A higher sampling rate of the measurements, i.e., a lower TR, in combination with higher magnetic field strengths may be important 

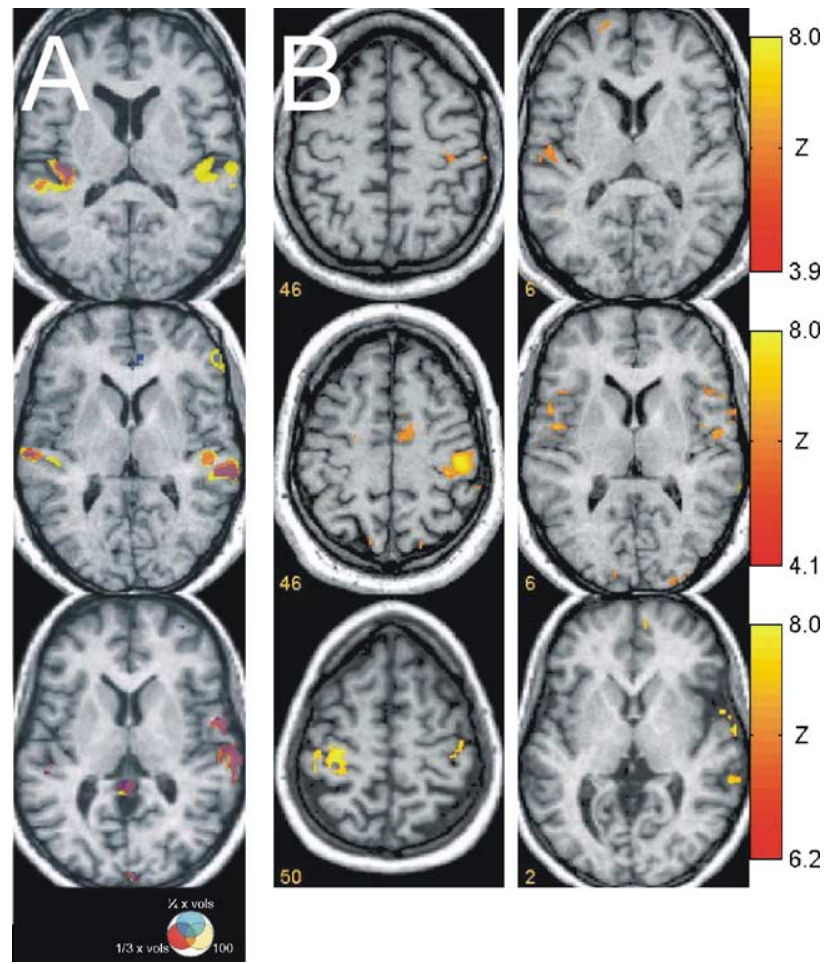

Fig. 6. (A) Degree of spatial overlap between auditory components of interest during hallucinations of patients $\mathrm{A}, \mathrm{D}$, and $\mathrm{E}$. Maps were thresholded at $Z=3$. Initial decomposition $=1 / 4 \times$ number of volumes (blue); additional decompositions $=1 / 3 \times$ number of volumes (red) and 100 independent components regardless of number of volumes (yellow). Orange and purple indicate overlap across decompositions. Left hemisphere is depicted on the left side. For patient A, bilateral Heschl's gyrus was found for the decomposition of 100 components. (B) Correlation maps of auditory hallucinations of the same patients, thresholded at the number of correlating voxels $=$ number of suprathreshold component of interest voxels. Only patients $\mathrm{A}$ and $\mathrm{E}$ show activity during hallucinations in Heschl's gyrus.

in order to catch more transient episodes. Furthermore, higher sampling rates allow for the analysis of temporal relations between cortical areas. Further studies are needed to investigate whether faster measurements, perhaps of only the auditory cortex, could improve detection of brain activity during hallucinations.

The current study is limited by several factors. First of all, the sample size was rather small, which is a general problem of fMRI studies with hallucinating psychiatric patients because only few of them are able or willing to report on their hallucinations while in the tomograph. Perhaps the use of data-driven analysis methods may help to alleviate the current necessity for patients to overtly respond to their hallucinations, which may facilitate patient recruitment in the future. The relatively small number of patients,

Table 5

Spatial correlations $(r)$ between initial decomposition (1) of $1 / 4 \times$ number of volumes and additional decompositions $(2$ and 3$)$ of $1 / 3 \times$ number of volumes and 100 components regardless of number of volumes

\begin{tabular}{llll}
\hline Patient & A & D & E \\
\hline$r_{1,2}$ & 0.53 & 0.66 & 0.79 \\
$r_{1,3}$ & $0.32^{*}$ & 0.73 & 0.75 \\
\hline
\end{tabular}

* Component of 3rd decomposition comprises bilateral Heschl's gyrus at threshold of $Z=3$.
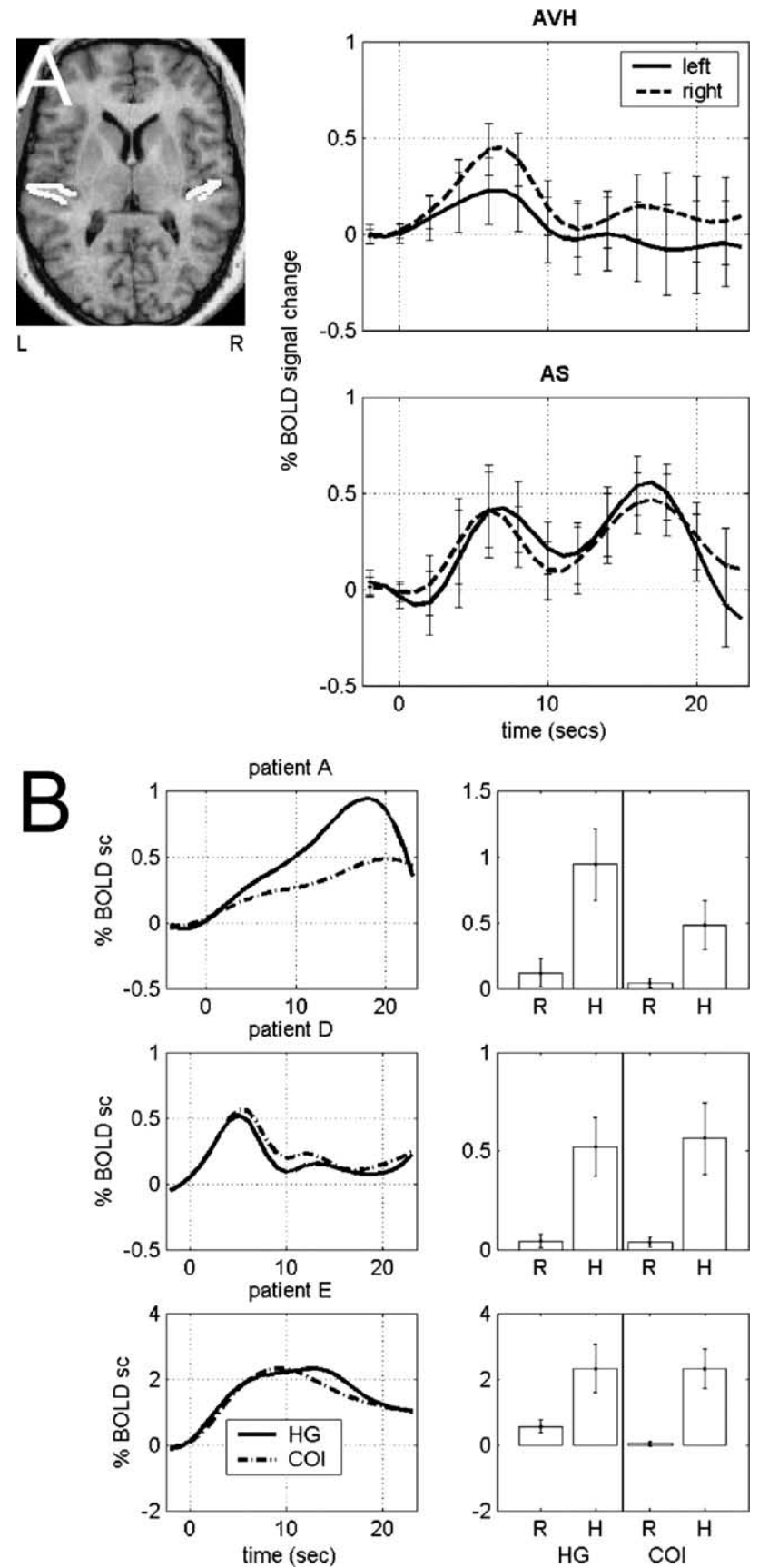

Fig. 7. (A) Event-related averages of activity in left and right Heschl's gyrus (delineated in white in the anatomical image) during hallucinations (AVH) and acoustic stimulation (AS) of patient D. Average time courses have similar shapes to the BOLD-image averages for AVH (Fig. 2) and AS (Fig. 4). (B) Event-related plots (left) of the COI activity time course of the whole component (COI) and of clusters in left and right Heschl's gyrus (HG) of patients A, D, and E. Bar charts (right) show mean and standard error of the peak amplitude. $\mathrm{HG}=$ Heschl's gyrus; $\mathrm{COI}=$ component of interest; $\mathrm{R}=$ no hallucination; $\mathrm{H}=$ during hallucinations.

difference in design parameters across patients, and interindividual variability make the generalization of our findings to the schizophrenia population as a whole impossible. Furthermore, we did not use specialized methods to exactly identify and analyze tonotopic primary auditory cortical fields (Formisano et al., 2003), and therefore cannot exclude that our findings partly reflect 
physiological processes unrelated to PAC. Finally, our results may be influenced by limitations of sICA. It is not known if the assumption of ICA, which states that the observed data is a linear combination of sources, is completely accurate for fMRI data and how the decomposition is affected if this assumption does not hold. Also, the choice of the number of components for a decomposition is of particular importance. Underestimating the number of sources in the data may result in an erroneous decomposition of the data, while overestimation may result in artifactual separation of one source across multiple components (Formisano et al., 2004). We decomposed the individual data sets using three different choices for number of components, and found that the COI maps remained largely stable across decompositions. A region of interest analysis of bilateral Heschl's gyrus of patient D confirmed the corresponding COI of bilateral auditory cortex activity during hallucinations. Therefore, it is not likely that our results are compromised by a faulty decomposition of the data structure. It should be noted, however, that any interpretation of sICA maps has to be given with caution because the method, without any further assumptions about the nature of the signals and the noise, does not provide any measure of probability of error, nor does it assess the functional significance of the signal of interest. However, this potential drawback has the benefit of revealing unpredicted patterns in the data, on which basis new hypotheses can be generated.

In conclusion, by applying sICA to fMRI data we showed brain activity in the auditory cortex, including PAC, of both hemispheres during AVH in a subsample of patients. The activity patterns reveal a large temporal variability, which makes the development of an appropriate predefined model of brain activity very complicated. Whether auditory cortex activity can be detected during hallucinations may be related to the length of the hallucination episodes. SICA has the potential to identify these activity patterns without the necessity of any model of activity, which makes it a very powerful tool for the analysis of neurophysiological signals that are related to the subjective experience of psychiatric patients.

\section{Acknowledgments}

This study was financially supported by the Vigoni programme of the German Academic Exchange service.

Prof. K. Maurer, head of the Department of Psychiatry of Frankfurt University, is gratefully acknowledged for constant support. Prof. F. Zanella and Dr. H. Lanfermann of the Department of Neuroradiology of Frankfurt University kindly provided access to the MR tomograph. The authors would like to extend their particular thanks to the patients who participated in the study.

\section{References}

American Psychiatric Association, 1994. Diagnostic and Statistical Manual of Mental Disorders, 4th ed. American Psychiatric Press, Washington, DC.

Bandettini, P.A., Jesmanowicz, A., Wong, E.C., Hyde, J.S., 1993. Processing strategies for time-course data sets in functional MRI of the human brain. Magn. Reson. Imaging 30, 161-173.

Bandettini, P.A., Jesmanowicz, A., Van Kylen, J., Birn, R.M., Hyde, J.S., 1998. Functional MRI of brain activation induced by acoustic scanner noise. Magn. Reson. Med. 39, 410-416.

Bentaleb, L.A., Beauregard, M., Liddle, P., Stip, E., 2002. Cerebral activity associated with auditory verbal hallucinations: a functional magnetic resonance imaging case study. J. Psychiatry Neurosci. 27, $110-115$.

Blakemore, S.J., Smith, J., Steel, R., Johnstone, C.E., Frith, C.D., 2000. The perception of self-produced sensory stimuli in patients with auditory hallucinations and passivity experiences: evidence for a breakdown in self-monitoring. Psychol. Med. 30, 1131-1139.

Boynton, G.M., Engel, S.A., Glover, G.H., Heeger, D.J., 1996. Linear systems analysis of functional magnetic resonance imaging in human V1. J. Neurosci. 16, 4207-4211.

Calhoun, V.D., Adali, T., Pearlson, G.D., Pekar, J.J., 2001. A method for making group inferences from functional MRI data using independent component analysis. Hum. Brain Mapp. 14, 140-151.

Calhoun, V.D., Kiehl, K.A., Liddle, P.F., Pearlson, G.D., 2004. Aberrant localization of synchronous hemodynamic activity in auditory cortex reliably characterizes schizophrenia. Biol. Psychiatry 55, $842-849$.

Dierks, T., Linden, D.E.J., Jandl, M., Formisano, E., Goebel, R., Lanfermann, H., Singer, W., 1999. Activation of Heschl's gyrus during auditory hallucinations. Neuron 22, 615-621.

Duann, J.-R., Jung, T.-P., Kuo, W.-J., Yeh, T.-C., Makeig, S., Hsieh, J.-C., Sejnowski, T.J., 2002. Single-trial variability in event-related BOLD signals. Neuroimage 15, 823-835.

Engelien, A., Huber, W., Silbersweig, D., Stern, E., Frith, C.D., Döring, W., et al., 2000. The neural correlates of 'deaf-hearing' in man: conscious sensory awareness by attentional modulation. Brain 123 , $532-545$.

Ffytche, D.H., Howard, R.J., Brammer, M.J., David, A., Woodruff, P., Williams, S., 1998. The anatomy of conscious vision: an fMRI study of visual hallucinations. Nat. Neurosci. 1, 738-742.

Formisano, E., Esposito, F., Kriegeskorte, N., Tedeschi, G., Di Salle, F., Goebel, R., 2002. Spatial independent component analysis of functional magnetic resonance imaging time-series: characterization of the cortical components. Neurocomputing 49, 241-254.

Formisano, E., Kim, D.-S., Di Salle, F., Van de Moortele, P.-F., Ugurbil, K., Goebel, R., 2003. Mirror-symmetric tonotopic maps in human primary auditory cortex. Neuron 40, 859-869.

Formisano, E., Esposito, F., Di Salle, F., Goebel, R., 2004. Cortexbased independent component analysis. Magn. Reson. Imaging 22, $1493-1504$.

Gu, H., Engelien, W., Hanhua, F., Silbersweig, D.A., Stern, E., Yang, Y., 2001. Mapping transient, randomly occurring neuropsychological events using independent component analysis. Neuroimage 14, $1432-1443$.

Halpern, A.R., Zatorre, R.J., 1999. When that tune runs through your head: a PET investigation of auditory imagery of familiar melodies. Cereb. Cortex 9, 697-704.

Harms, M.P., Melcher, J.R., 2003. Detection and quantification of a wide range of fMRI temporal responses using a physiologically-motivated basis set. Hum. Brain Mapp. 20, 168-183.

Hirayasu, Y., McCarley, R.W., Salisbury, D.F., Tanaka, S., Kwon, J.S., Frumin, M., Snyderman, D., Yurgelon-Todd, D., Kikinis, R., Jolesz, F.A., Shenton, M., 2002. Planum temporale and Heschl gyrus volume reduction in schizophrenia. Arch. Gen. Psychiatry 57, 692-699.

Hubl, D., Koenig, T., Strik, K., Federspiel, A., Kreis, R., Boesch, C., Maier, S.E., Schroth, G., Lovblad, K., Dierks, T., 2004. Pathways that make voices: white matter changes in auditory hallucinations. Arch. Gen. Psychiatry 61, 658-668.

Hyvärinen, A., 1999. Fast and robust fixed-point algorithms for independent component analysis. IEEE Trans. Neural Netw. 10, 626-634.

Jäncke, L., Shahram, M., Shah, N.J., 1999. Attention modulates activity in the primary and the secondary auditory cortex: a functional magnetic resonance imaging study in human subjects. Neurosci. Lett. 266, $125-128$.

Jung, T.-P., Makeig, S., Westerfield, M., Townsend, J., Courchesne, E., Sejnowski, T.J., 2001. Analysis and visualization of single-trial eventrelated potentials. Hum. Brain Mapp. 14, 166-185.

Lennox, B.R., Park, S.B., Jones, P.B., Morris, P.G., Park, G., 1999. Spatial 
and temporal mapping of neural activity associated with auditory hallucinations. Lancet 353, 644

Lennox, B.R., Park, S.B., Medley, I., Morris, P.G., Jones, P.B., 2000. The functional anatomy of auditory hallucinations in schizophrenia. Psychiatry Res. 100, 13-20.

McKeown, M.J., Makeig, S., Brown, G.G., Jung, T.-P., Kindermann, S.S., Bell, A.J., Sejnowski, T.J., 1998a. Analysis of fMRI data by blind separation into independent spatial components. Hum. Brain Mapp. 6 , $160-188$.

McKeown, M.J., Jung, T.-P., Makeig, S., Brown, G., Kindermann, S.S., Lee, T.-W., Sejnowski, T.J., 1998b. Spatially independent activity patterns in functional MRI data during the Stroop color-naming task. Proc. Natl. Acad. Sci. U. S. A. 95, 803-810.

Rademacher, J., Morosan, P., Schormann, T., Schleicher, A., Werner, C. Freund, H.-J., Zilles, K., 2001. Probabilistic mapping and volume measurement of human primary auditory cortex. Neuroimage 13, 669-683.

Seifritz, E., Esposito, F., Hennel, F., Mustovic, H., Neuhoff, J.G., Bilecen, D., et al., 2002. Spatiotemporal pattern of neural processing in the human auditory cortex. Science 297, 1706-1708.

Shergill, S.S., Brammer, M.J., Williams, S.C., Murray, R.M., McGuire, P.K., 2000. Mapping auditory hallucinations in schizophrenia using functional magnetic resonance imaging. Arch. Gen. Psychiatry 57, $1033-1038$

Shergill, S.S., Bullmore, E.T., Brammer, M.J., Williams, S.C.R., Murray,
R.M., McGuire, P.K., 2001. A functional study of auditory verbal imagery. Psychol. Med. 31, 241-253.

Silbersweig, D.A., Stern, E., Frith, C., Cahill, C., Holmes, A., Grootoonk, S., et al., 1995. A functional neuroanatomy of hallucinations in schizophrenia. Nature 378, 176-179.

Stephane, M., Barton, S., Boutros, N.N., 2001. Auditory verbal hallucinations and dysfunction of the neural substrates of speeches. Schizophr. Res. 50, 61-78.

Stephane, M., Thuras, P., Nasrallah, H., Georgopoulos, A.P., 2003. The internal structure of the phenomenology of auditory verbal hallucinations. Schizophr. Res. 61, 185-193.

Talairach, J., Tournoux, P., 1988. Co-Planar Stereotaxic Atlas of the Human Brain. Thieme Medical Publisher, New York.

Van de Ven, V.G., Formisano, E., Prvulovic, D., Roeder, C.H., Linden, D.E.J., 2004. Functional connectivity as revealed by spatial independent component analysis of fMRI measurements during rest. Hum. Brain Mapp. 22, 165-178.

Wheeler, M.E., Petersen, S.E., Buckner, R.L., 2000. Memory's echo: vivid remembering reactivates sensory-specific cortex. Proc. Natl. Acad. Sci. U. S. A. 20, 11125-11129

Woodruff, P.W.R., Wright, I.C., Bullmore, E.T., Brammer, M., Howard, R.J., Williams, S.C., 1997. Auditory hallucinations and the temporal cortical response to speech in schizophrenia: a functional magnetic resonance imaging study. Am. J. Psychiatry 154, 1676-1682. 